\title{
Toward a Bandwidth Efficient Cognitive Positioning System
}

\author{
R. R. Thomas, Student Member IEEE, B. T. Maharaj, Member IEEE, Member IET
}

Presented is an efficient bandwidth determination model for the Cognitive Positioning System by utilising multiple receive antennas. The performance evaluations of the proposed multiple receive antenna positioning system were carried out using the linear least squares and maximum-likelihood location estimation techniques. The simulated results reveal that in a rural scenario, the $1 \times 2$ and $1 \times 3$ single-input-multiple-output (SIMO) cases display an average bandwidth efficiency with respect to positional accuracy, of $49 \%$ and $58 \%$ respectively, over the single-input-single-output (SISO) case. For an urban scenario, the $1 \times 2$ and $1 \times 3$ SIMO cases display a higher average bandwidth efficiency of $53 \%$ and $62 \%$ respectively over the SISO case.

Introduction: A key aspect which has become the focus of current research is the location and environment awareness capability of cognitive radio [1, 2]. Utilisation of location information forms an important part of cognitive radio and therefore integral to the IEEE 802.22 working standard for Wireless Regional Area Networks (WRAN) [3]. One such model which has been referred to as the Cognitive Positioning System (CPS) [4] addresses the location awareness and adaptive requirements of cognitive radio using dynamic spectrum access techniques. The authors propose an optimization of the existing CPS by increasing the bandwidth efficiency using a single-input-multiple-output (SIMO) antenna positioning system. Using the Cramer-Rao lower bound (CRLB) principle, an appropriate theoretical bandwidth determination model is derived and compared to the conventional single-input-single-output (SISO) case using the linear least squares (LLS) 
and maximum-likelihood (ML) estimation algorithms under generic rural and urban environmental models.

CPS Bandwidth Determination Signal Model: The CPS consists of two interdependent tasks, viz. Bandwidth Determination (BD) and Enhanced Dynamic Spectrum Management (EDSM) [4], which provide adaptive positioning capabilities suited for cognitive radio. The BD model ascertains the amount of bandwidth required to obtain a predefined positional accuracy using the time-of-arrival (TOA) ranging technique. The EDSM component determines in real-time whether the required bandwidth from the BD model is available in the spectrum using dynamic spectrum access techniques. Let the signal model consist of a single input antenna with $N$ equally spaced output antennas operating in a single-path additive white Gaussian noise (AWGN) channel. Accordingly a generalised narrowband received signal can be mathematically modelled as:

$$
r_{l}=\alpha_{l} s\left(t-\tau_{o}\right)+n_{l}(t), 0 \leq t \leq T
$$

where $l$ represents the number of antennas from $l=1, \ldots, N, \alpha_{l}$ is the complex channel coefficient of each antenna, $s(t)$ represents the original transmitted signal band-limited to $\mathrm{B} \mathrm{Hz}, \tau_{o}$ is the path delay and $n_{l}(t)$ represents AWGN with zero mean and spectral density of $\sigma_{l}^{2}$. It has been assumed that the signal delays $\tau_{o}$ for the different antennas are identical as this study involves a single-path case. According to (1), $\alpha_{l}$ and $\tau_{o}$ are key

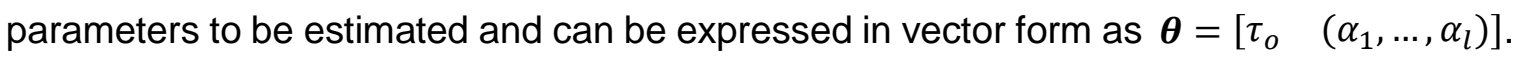
The observation interval encompasses the symbol time $\left(T_{s}\right)$, number of transmitted symbols $(M)$ and maximum time delay $\left(\tau_{o_{\max }}\right)$ and is shown as: $T=M T_{s}+\tau_{o_{\max }}$. The continuous waveform given by (1) is sampled at the Nyquist rate and is given by: $T_{\text {samp }}=\frac{1}{2 B}$. The elements of the $2 \times 2$ Fisher Information Matrix (FIM) have been determined for a general Gaussian case for a discrete received signal using [5]: 


$$
[\boldsymbol{I}(\theta)]_{i j}=\sum_{l=1}^{N} \frac{1}{\sigma_{l}^{2}} \sum_{k=0}^{K-1} \frac{\partial s[k ; \theta]}{\partial \theta_{i}} \frac{\partial s[k ; \theta]}{\partial \theta_{j}},
$$

where $i=1,2, \ldots, q, j=1,2, \ldots, q$ and $l$ and $k$ represent the number of antennas and number of discrete samples respectively. It has also been assumed that the spectral density $\left(\sigma_{l}^{2}\right)$ across all receiver branches is the same. Using (2), the following two FIM elements have been determined:

$$
\begin{aligned}
I_{\tau \tau} & =\left(\hat{\varepsilon}-\frac{\tilde{\varepsilon}^{2}}{\varepsilon}\right) \sum_{l=1}^{N} \frac{\left|\alpha_{l}\right|^{2}}{\sigma_{l}^{2} T_{\text {samp }}}, \\
\boldsymbol{I}_{\boldsymbol{\alpha} \boldsymbol{\alpha}} & =\frac{\varepsilon}{T_{\text {samp }}} \times \operatorname{diag}\left[\frac{1}{\sigma_{1}^{2}}, \ldots, \frac{1}{\sigma_{l}^{2}}\right] .
\end{aligned}
$$

The inverse matrix of (3) and (4) represent the CRLB of the time delay and channel coefficents respectively. Hence:

$$
\begin{aligned}
& \operatorname{var}\left(\widehat{\tau_{o}}\right)=\frac{1}{\left(\hat{\varepsilon}-\frac{\tilde{\varepsilon}^{2}}{\varepsilon}\right) \sum_{l=1}^{N} \frac{\left|\alpha_{l}\right|^{2}}{\sigma_{l}^{2} T_{\text {samp }}},} \\
& \operatorname{var}(\widehat{\boldsymbol{\alpha}})=D_{i j}=\left\{\begin{array}{c}
\frac{\varepsilon_{i}-\frac{\tilde{\varepsilon}^{2} \sum_{l=1}^{N} \frac{\left|\alpha_{l}\right|^{2}}{\left(\sigma_{l}^{2}\right)^{2}}}{\sigma_{i}^{2} T_{\text {samp }}}-\frac{\hat{\varepsilon} T_{\text {samp }} \sum_{l=1}^{N} \frac{\left|\alpha_{l}\right|^{2}}{\sigma_{l}^{2}}}{\tilde{\varepsilon}^{2} \sum_{l=1}^{N}\left(\frac{\left|\alpha_{l}\right|^{2}}{\sigma_{l}^{2} T_{\text {samp }}}\right)^{2}}}{-\frac{\left|\alpha_{l}\right|^{2}}{\sigma_{l}^{2}}} \quad i \neq j
\end{array},\right.
\end{aligned}
$$

where $\operatorname{var}\left(\widehat{\tau_{o}}\right)$ is the variance of the time delay estimate, $\operatorname{var}(\widehat{\boldsymbol{\alpha}})$ is the variance of the channel coefficient estimates, $\hat{\varepsilon}=M \int_{0}^{T_{s}}\left|s^{\prime}\left(t-\tau_{o}\right)\right|^{2} d t$ and $\tilde{\varepsilon}=M \int_{0}^{T_{s}}\left|s^{\prime}\left(t-\tau_{o}\right)\right|\left|s\left(t-\tau_{o}\right)\right| d t$, while the energy of the signal over symbol time $T_{S}$ is given as:

$$
\varepsilon=M \int_{0}^{T_{s}}\left|s\left(t-\tau_{o}\right)\right|^{2} d t
$$


The derivative of the signal energy is shown as $s^{\prime}\left(t-\tau_{o}\right)$. It also assumed that a priori information about the channel coefficients are available. The bandwidth representation in the frequency domain can be given by $\beta^{2}=\frac{\int_{-\infty}^{\infty} f^{2}|S(f)|^{2} d f}{\int_{-\infty}^{\infty}|S(f)|^{2} d f}$, where the Fourier Transform of $s(t)$ is shown as $S(f)$ and therefore using (7) one can express $\hat{\varepsilon}$ as:

$$
\hat{\varepsilon}=\varepsilon \beta^{2} \text {. }
$$

The positional accuracy $(P(\hat{d}))$ is defined as (where $\hat{d}$ is the estimated distance):

$$
P(\hat{d})=\frac{1}{c^{2} \operatorname{var}\left(\hat{\tau_{o}}\right)}
$$

where the electromagnetic wave speed $(c)$ is taken to be $3 \times 10^{8} \mathrm{~m} / \mathrm{s}$. Therefore using the results from (5),(8) and (9) the proposed efficient bandwidth determination model for a general narrowband signal $s(t)$ is shown to be:

$$
\hat{\beta}=\sqrt{\frac{c^{2} P(\hat{d})}{M \gamma_{s} \sum_{l=1}^{N}\left|\alpha_{l}\right|^{2}}} .
$$

The (signal-to-noise ratio) SNR is given by $\gamma_{S}=\frac{\varepsilon}{N_{o}}$, (where $N_{o}$ is the noise power). According to the derivation in (10), the accuracy of the required bandwidth $(\hat{\beta})$ for performing adaptive TOA positioning is primarily affected by the SNR and channel coefficients at each receive antenna.

Results \& Discussion: In order to evaluate the performance of the the proposed SIMO BD model, a comparison of the bandwidth efficiency with respect to the positional root mean square error (RMSE) was drawn against a conventional SISO system over a predefined bandwidth interval ranging from $1 \mathrm{MHz}$ to $20 \mathrm{MHz}$. The $1 \times 2$ and $1 \times 3$ SIMO BD positioning model was analysed using the LLS and ML estimation technique at a fixed SNR of $10 \mathrm{~dB}$. Fig. 1 and Fig. 2 represent the mobile terminal's (MT's) two-dimensional (2D) positioning RMSE as a function of utilized bandwidth in a generalised rural and urban scenario, 
respectively. Both results indicate that diversity for multiple antenna positioning systems plays an important role in improving bandwidth efficiency. For the rural and more especially the urban case a significant performance advantage in terms of positional accuracy of the SIMO positioning scheme can be attained at lower bandwidth intervals from $1 \mathrm{MHz}$ to $8 \mathrm{MHz}$. This can enable improved dynamic spectrum access for positioning applications in cognitive radio, more particularly in cases where there is an absence of large discrete bandwidths. It was observed that for bandwidths beyond $20 \mathrm{MHz}$, the SIMO positional RMSE for both the $1 \times 2$ and $1 \times 3$ scheme converge at an average RMSE of $0.1 \mathrm{~m}$ for the rural case and $0.2 \mathrm{~m}$ for the urban case, which indicate a minimal change in positional accuracy as the bandwidth is increased. Furthermore, it has been determined that the utilisation of more than three receive antennas produced a minimal differential change of approximately $10 \%$ in the positional RMSE when compared to the $1 \times 3$ case for both types of environments. The performance evaluations therefore show that the $1 \times 3$ case was deemed to be the optimal SIMO scheme in terms of achieving improved bandwidth efficiency for the CPS. The ML and LLS estimation techniques for the rural scenario display similar performace in terms of positional accuracy. According to Fig. 2, in the case of a typical urban scenario, the LLS estimation technique is susceptible to more variations in positional accuracy than the ML techinque in the presence of non-line-of-sight (NLOS) signal paths for both SIMO schemes. However the ML technique is computationally more complex than the LLS technique, due to the addition of nosie statistics in the algorithm. This result indicates that the CPS could adaptively select the estimation technique with greater stability or least complexity according to the localisation application requirement in this type of enivironment.

Conclusion: The comparative bandwidth efficiency of both the SISO and SIMO bandwidth determination models for the Cognitive Positioning System have been presented for two 
typical scenarios. The simulation results highlight the advantage of implementing multiple antennas and hence the role of spatial diversity at the cognitive radio receiver for improving bandwidth efficiency in order to enable optimised dynamic spectrum access with respect to location awareness.

\section{References}

1. CELEBI, H., and ARSLAN, H.: 'Enabling location and environment awareness in cognitive radios', Comp. Commun., 2008, 31, (4) pp. 1114-1125

2. CELEBI, H., and ARSLAN, H.: 'Utilisation of location information in Cognitive Wireless Networks', IEEE Wireless Commun. Mag., 2007, 14, (4), pp. 6-13

3. STEVENSON, C.R., CHOUINARD, G., LEI, Z., HU, W., SHELLHAMMER, S.J., and CALDWELL, W.: 'IEEE 802.22 The First Cognitive Radio Wireless Regional Area Network Standard, IEEE Commun. Mag., 2009, 47, (1), pp. 130-138

4. CELEBI, H., and ARSLAN, H.: 'Cognitive Positioning Systems', IEEE Trans. Wireless Commun., 2007, 6, (12), pp. 4475-4483

5. KAY, SM.: 'Fundamentals of Statistical Signal Processing Volume 1: Estimation Theory', (Addison Wesley Longman, 2001)

\section{Authors' affiliations:}

R.R. Thomas and B.T. Maharaj are from the Department of Electrical, Electronic and Computer Engineering, University of Pretoria, Pretoria, 0002, South Africa, e-mail: robinthomas@ieee.org.

\section{Acknowledgements}

This research work was financially supported by the Sentech Chair in Broadband Wireless Multimedia Communications at the University of Pretoria and the National Research Foundation. 


\section{List of Figures}

Figure 1: RMSE of the positional accuracy for the SISO and SIMO schemes in a typical rural environment.

$\square \quad$ SISO Implementation of ML Estimation

- SISO Implementation of LLS Estimation

$\longrightarrow \quad$ SIMO Implementation of ML Estimation Estimation with 2 receive antennas

$\longrightarrow$ SIMO Implementation of LLS Estimation Estimation with 2 receive antennas

— SIMO Implementation of ML Estimation Estimation with 3 receive antennas

SIMO Implementation of LLS Estimation Estimation with 3 receive antennas

Figure 2: RMSE of the positional accuracy for the SISO and SIMO schemes in a typical urban environment.

$\square \quad$ SISO Implementation of ML Estimation

- SISO Implementation of LLS Estimation

$\longrightarrow \quad$ SIMO Implementation of ML Estimation Estimation with 2 receive antennas

$\longrightarrow$ SIMO Implementation of LLS Estimation Estimation with 2 receive antennas

— SIMO Implementation of ML Estimation Estimation with 3 receive antennas

SIMO Implementation of LLS Estimation Estimation with 3 receive antennas 
Figure 1

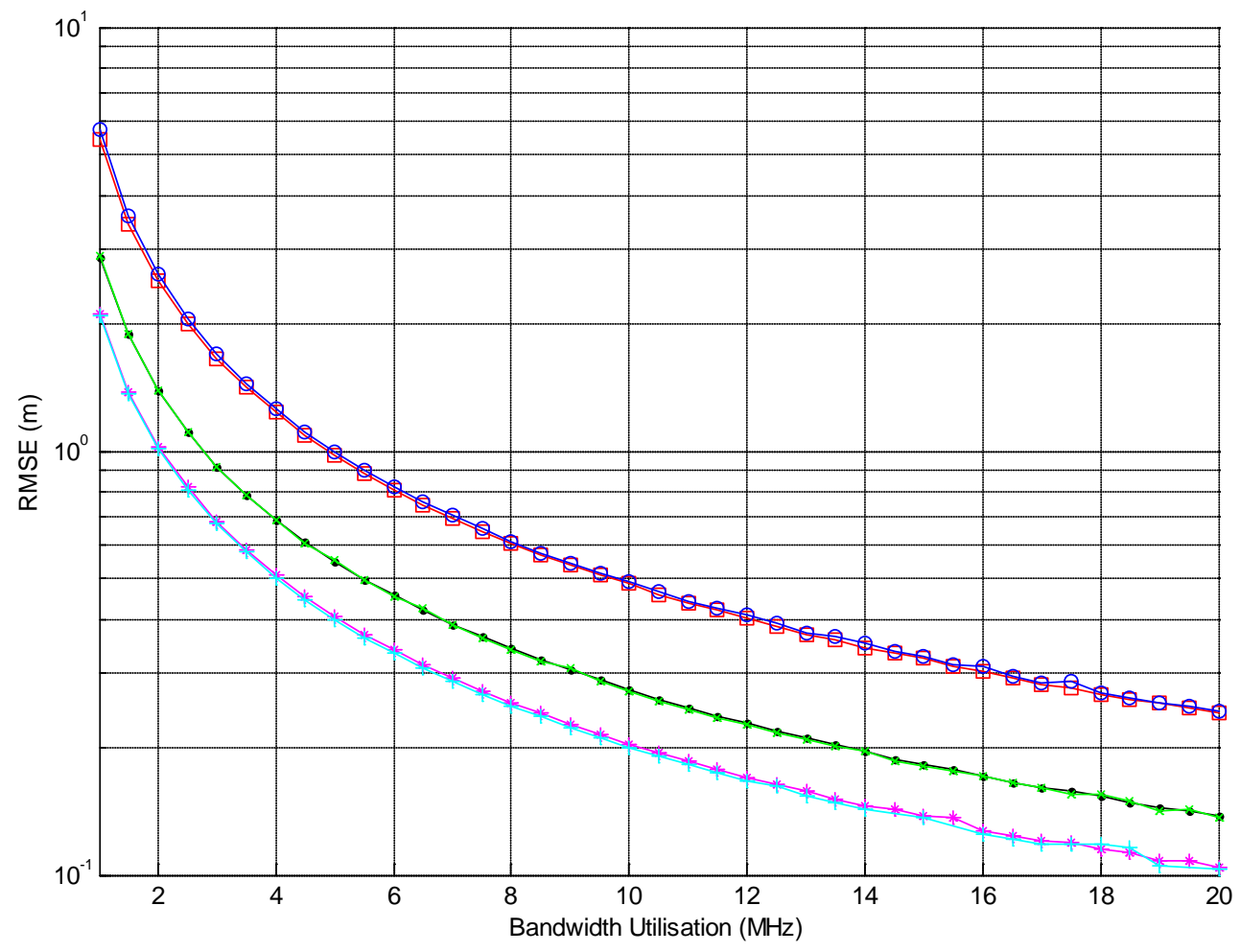


Figure 2

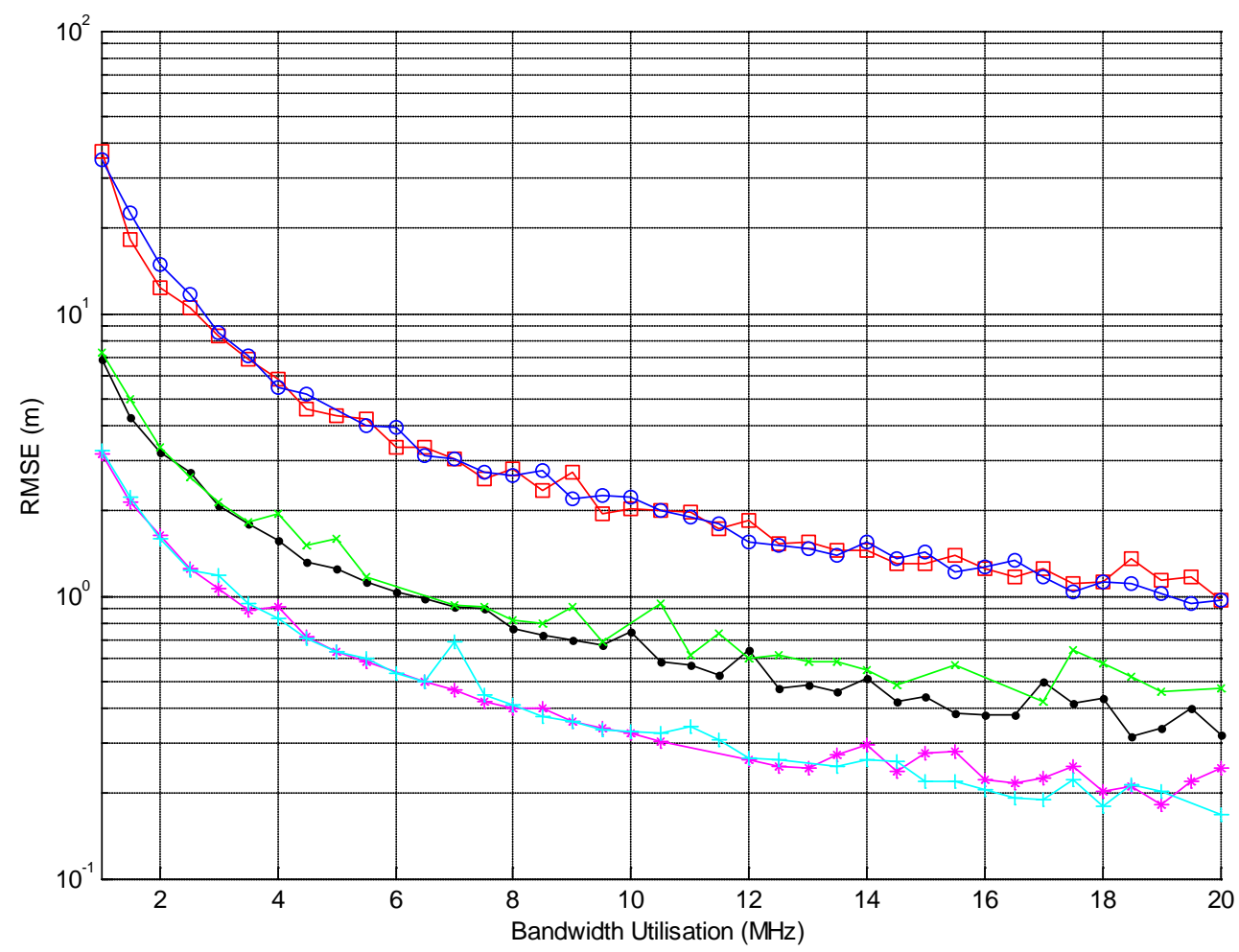

